\title{
Funcionamiento familiar según el modelo Circumplejo de Olson en familias con un niño que presenta retardo mental
}

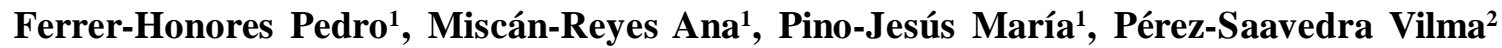

\section{RESUMEN}

Objetivo: conocer el funcionamiento familiar en familias que tienen un niño con retardo mental, según la teoría de David Olson, a través de la dimensión de adaptabilidad y cohesión, el tipo de funcionamiento familiar, entre las cuales están el balanceado, medio y externo. Material y métodos: tipo descriptivo - transversal con una población total de 42 cuidadores de niños con diagnóstico médico de retardo mental, que estudian en la Institución educativa especial Divina Misericordia en el distrito de Villa El Salvador, en el año escolar 2010. Para la recolección de datos se aplicó un cuestionario para obtener las características socioeconómicas familiares, compuesto por 7 preguntas, y la escala de evaluación familiar FACES III, diseñado y aplicado por David Olson, compuesto por 20 preguntas, 10 de las cuales pertenecen a la dimensión de cohesión y las 10 restantes pertenecen a la dimensión de adaptabilidad. Los cuestionarios fueron respondidos por las madres de los niños con retardo mental. El programa escogido para el procedimiento de datos del estudio fue Excel V.2007 de Windows Vista. Resultados: Los resultados obtenidos indican que casi la mitad de la población estudiada (48\%), muestra un funcionamiento familiar de rango medio. Según cohesión se observó que el mayor porcentaje de las familias pertenecía a cohesión media, siendo familias semirrelacionada en un total de $38 \%$. Según adaptabilidad se obtuvo que el $42.9 \%$ de las familias son altamente caóticas. Conclusiones: El funcionamiento familiar en familias que tienen un niño con retardo mental es de rango medio. Con respecto al funcionamiento familiar según la cohesión la población presentó un nivel medio A, la cual representa a familias tipo semirrelacionadas. Según adaptabilidad se observó que el mayor porcentaje de las familias resultaron caóticas.

Palabras clave: familia, funcionalidad, adaptabilidad. Perú (Fuente DeCs BIREME).

\section{Family functioning as the Olson Circumplex model in families with a child with mental retardation}

\begin{abstract}
Objetive: Meet family functioning in families with a child with mental retardation, according to the theory of David Olson, through the dimension of adaptability and cohesion, the type of family functioning, among which are the balanced, middle and outer. Material and Methods: Descriptive - cross with a total population of 42 caregivers of children with a medical diagnosis of mental retardation, studying in the special Divine Mercy Educational Institution in the district of Villa El Salvador, in the 2010 school year for data collection was applied a questionnaire for family socioeconomic characteristics, consisting of 7 questions, and family assessment scale FACES III, designed and implemented by David Olson, composed of 20 questions, 10 of which belong to the dimension of cohesion and the remaining 10 are the dimension of adaptability. The questionnaires were answered by mothers of children with mental retardation. The method chosen for the program of study data was Excel v.2007 for Windows Vista. Results: The results indicate that almost half of the study population (48\%) shows a midrange family functioning. According cohesion was observed that the highest percentage of families belonged to average cohesion being semirrelacionada families in a total of $38 \%$. According adaptability was obtained $42.9 \%$ of families are highly chaotic. Conclusions. Family functioning in families
\end{abstract}

Licenciada en Enfermería. Facultad de Enfermería de la Universidad Peruana Cayetano Heredia. Lima-Perú

Docente de la Facultad de Enfermería de la Universidad Peruana Cayetano Heredia. Lima-Perú 
who have a child with mental retardation is midrange. With respect to family functioning as cohesion population had a mean level A, which represents families semirrelacionadas type. According adaptability was observed that the highest percentage of families were chaotic.

Key words: Family, functioning, adaptability. Peru (Source DeCs BIREME).

\section{INTRODUCCIÓN}

La familia, unidad básica de la sociedad, base importante para el desarrollo humano, es definida como «la unión de personas que comparten un proyecto vital de existencia común, en el que se generan fuertes sentimientos de pertenencia, existe un compromiso personal entre sus miembros y se establecen intensas relaciones de intimidad, reciprocidad y dependencia (1); es la encargada de brindar los cuidados básicos de bienestar y salud a sus miembros. Esto la convierte en el factor más importante para el desarrollo emocional, físico y social del niño. Pero para que la familia pueda lograr este propósito debe cumplir con sus funciones, lo cual la llevará a mantener un funcionamiento familiar saludable (2).

La familia es para el niño con retardo mental, el contexto en donde se dan las primeras condiciones para el desarrollo favorable y saludable de su personalidad así como su desarrollo social. Para el logro de este papel, la familia debe cumplir adecuadamente con sus funciones, a lo que se llama funcionamiento familiar (1). Una familia saludable en todas sus relaciones y aspectos, así como también la estabilidad de cada uno de sus miembros ayudarán a que el niño con retardo mental se desenvuelva en un ambiente adecuado y además mantenga buenas relaciones con sus familiares.

Para Olson, la definición del funcionamiento familiar es la interacción de vínculos afectivos entre miembros de la familia (cohesión) y que pueda ser capaz de cambiar su estructura con el fin de superar las dificultades evolutivas familiares (adaptabilidad) (11). Esta teoría contempla las situaciones que atraviesan la familia durante la vida y los cambios que deben realizar en su estructura para lograr una adaptación y una cohesión, que ayuden a una mejor estabilidad para el crecimiento saludable del niño con retardo mental. Además establece que un funcionamiento familiar balanceado es aquel que posibilita a la familia cumplir exitosamente con los objetivos y funciones que le están asignados. Muchas veces este funcionamiento puede ser adecuado, pero otras veces puede verse afectado por algunas situaciones o factores estresantes que se presenten a lo largo del ciclo evolutivo de la familia, como en el caso que tengan un niño con retardo mental que será considerado siempre como un discapacitado por su familia y la sociedad (4). El retardo mental es una de las discapacidades más comunes en el Perú. Se estima que el total de niños con retardo mental leve, moderado severo para el año 2007 a nivel nacional es de 3617 y para el año 2008 de Enero a Junio, es de 3272 desde 0 a 19 años. La concentración del mayor número de casos es en Lima. Para el año 2007 se encontró un total de 1800 y para el año 2008, de Enero a Junio, son 1597 entre 0 a 19 años (5).

Actualmente en nuestro país existe un gran número de familias que muchas veces no saben cómo actuar y desarrollar sus funciones para cuidar a su hijo con retardo mental ya sea por falta de conocimientos, problemas de afrontamiento o por falta de recursos económicos. Todo esto, sin duda, va a provocar que para la familia sea difícil tener y aceptar un hijo con retardo mental, es por eso que es natural que enfrenten sentimientos encontrados y de culpa, que generarán una disfunción familiar $(2,3)$.

La etiología del retraso mental se considera mayoritariamente multifactorial y la combinación de los diversos factores etiológicos condiciona la gran variedad de manifestaciones clínicas. Se acepta que aproximadamente en el 30-40\% (5) de estos casos, examinados a nivel de consulta externa, no se pueda determinar una etiología clara a pesar de haberlos sometido a complejas evaluaciones. En el resto de los casos, los principales factores causales se distribuyen de la siguiente forma: aproximadamente en un $50 \%$ se detecta factores hereditarios; en un 30\%, alteraciones tempranas del desarrollo embrionario; en un 50\%, trastornos somáticos de la niñez; mientras que las influencias del entorno y los trastornos mentales se registran entre el $15-20 \%$ de los casos. Estos factores no se excluyen entre sí (6).

Los niños al nacer con esta discapacidad quedan en manos de un cuidador para toda la vida, que en la mayoría de los casos es la madre. Ella y la familia en ocasiones van a proteger y amar al niño, pero otras veces va a ser rechazado y maltratado. Estas actividades irán cambiando el funcionamiento familiar, que se ve marcado por situaciones críticas que se añaden a las habituales en la relación familiar, y la forma de resolverlas condicionará los apoyos 
que se ofrezcan al niño con retardo mental (7).

En otros casos la situación en la que se encuentran no puede ser superada y los padres tienen sentimientos de culpa que propician que sientan miedo al enfrentar el repudio social y vergüenza de su hijo con retardo mental. Esta situación puede despertar problemas latentes, lo que generará cambios en el funcionamiento familiar. Además, se encuentra que en familias que tienen un niño con retardo mental, la necesidad de cohesión suele ser permanente, obstaculizando así los cambios de desarrollo normales de los miembros de la familia (7).

Pérez Martínez, desarrolló una investigación en Cuba titulada El impacto del déficit mental en el ámbito familiar, obteniendo como resultados que la repercusión del déficit mental para la salud familiar fue desfavorable en 89 familias y resultó favorable sólo para 17 núcleos familiares encuestados los que consideraron por consenso, que la discapacidad mental de uno de sus miembros contribuyó al crecimiento espiritual de los demás integrantes de la familia. Por esta razón se consideró que el miembro con déficit mental fue un motivador de cambios óptimos que ocurrieron en las relaciones afectivas entre los miembros de las familias afectadas (14).

Según la teoría de Olson, la familia según su funcionalidad se clasifica en: caótica, flexible, estructurada, rígida, disgregada, separada, relacionada y aglutinada. Para el estudio se trabajó con el Modelo Circumplejo de Olson; se observaron dos tipos de niveles que son cohesión y adaptabilidad. Dentro del Modelo Circumplejo los conceptos específicos para medir y diagnosticar la dimensión de cohesión son: vinculación emocional, límites, coalición, tiempo y espacio, amigos, toma de decisiones, Intereses y recreación y los conceptos específicos para diagnosticar y medir la dimensión de adaptabilidad son: estilo de negociación, poder en la familia (asertividad, control, disciplina), relaciones de roles, reglas de las relaciones.

La Cohesión se define como «el vinculo emocionales que los miembros de la familia tienen unos con otros y el grado de autonomía personal que experimentan.» Evalúa el grado por el cual los miembros están conectados o separados del resto de la familia. Esta dimensión se define por los vínculos emocionales que cada miembro tiene con el otro (4).

La adaptabilidad familiar según D. Olson, «se define como la habilidad de un sistema familiar para cambiar su estructura de poder, las relaciones de roles y las reglas de las relaciones, en respuesta al estrés situacional y propia del desarrollo», es decir que la estructura familiar puede verse afectada en un cambio, la desintegración de una familia nuclear (no logra la adaptabilidad el padre o la madre), hay cambios en las reglas y roles que los hijos muchas veces no están acostumbrados a realizar y para poder lograr esta habilidad tratan de adaptarse (11) .

La comunicación familiar es otra de las dimensiones importantes en este modelo. Es la tercera en el modelo Circumplejo de Olson, que se basa en la empatía, la escucha reflexiva. Esta dimensión está inmersa, actuando como facilitadora en las 2 primeras dimensiones, por tal motivo no se identifica como indicador de la evaluación en el FACES III (11).

La profesión de enfermería, sea en el nivel hospitalario o en la comunidad, tiene como uno de sus ejes de intervención a la famililla, como unidad básica, para brindar servicios de enfermería, considerando que el funcionamiento familiar afecta la salud de todos sus miembros (19).

Esta investigación, desde el punto de vista de la salud, atribuye un papel fundamental a la intervención de enfermería en la familia, contribuyendo de manera individual y colectiva en propiciar el nivel óptimo del usuario. Vista desde un enfoque holístico, la importancia del estudio radica en comprender el funcionamiento familiar de familias que tienen un niño con retardo mental, porque esta discapacidad puede ser considerado como un problema médico, pero se trata fundamentalmente de una problemática familiar ya que el paciente es un miembro de la familia que provocará transformaciones y cambios en el ámbito familiar, pero esencialmente es una persona que necesita la atención necesaria para obtener una buena calidad de vida que le permita un desarrollo como integrante de una familia y de una sociedad (3). La información que se obtendrá ayudará al personal de salud a determinar y analizar el funcionamiento familiar de estas familias y colaborar y brindar apoyo a las madres ante los cambios que puedan irse dando. Estas acciones contribuirán a un cuidado adecuado del niño que le facilitará el logro de una buena calidad de vida (2).

Además, durante nuestras prácticas pre profesionales en hospitales y la comunidad, observamos que eran las madres, como cuidadoras, las que más acudían a consulta con los niños con retardo mental o eran las que se encargaban de su cuidado en casa, encontrándose a veces sobrecargadas de estrés, y nos preguntábamos si sólo ellas formaban parte del ambiente familiar del niño ¿Dónde está el padre?, ¿Apoya en el cuidad? ¿Dónde están los hermanos? Por ello se quería determinar cómo funcionaba la 
familia en casa, sus roles y relaciones lo cual contempla David Olson en su teoría, en las dimensiones de cohesión y adaptabilidad.

El propósito de esta investigación radica sobre todo en conocer y comprender cómo es el funcionamiento familiar en las familias que tienen un niño con retardo mental, porque conocer esa realidad ayudará al personal de enfermería a desarrollar acciones para contribuir a la adaptación de la familia, a lo largo de su recorrido con un niño dependiente de ellos, y al fortalecimiento de sus relaciones, para que puedan cumplir con brindarle seguridad, protección y realización, evitando de esta manera que el niño con retraso mental quede sin apoyo y amor familiar (12). Por lo expuesto se decidió establecer la siguiente pregunta de investigación:

¿Cómo es el funcionamiento familiar, según el modelo Circumplejo de Olson, de familias que tienen un niño con retardo mental, estudiando la I.E.E «Divina Misericordia» del distrito de Villa El Salvador durante el año 2010?

\section{MATERIAL Y MÉTODOS}

El estudio fue tipo descriptivo, de corte transversal. La población estuvo conformada por las madres de los 42 niños con retardo mental, entre 5 y 11de edad, que estudian del primer al sexto grado de primaria en la institución educativa especial Divina Misericordia, ubicado en el distrito de Villa El Salvador.

Para recolectar la información se usaron un cuestionario que valora las características familiares y una escala de evaluación de la adaptabilidad y cohesión familiar faces iii (Olson, 1986, 1991).

Cuestionarios de autoaplicacion, validados y que fueron previamente explicados a los familiares para que conozcan la finalidad del estudio y participen voluntariamente, firmando el consentimiento informado.

Una vez obtenida la información, se obtuvo el puntaje del instrumento aplicado, sumando el puntaje de las preguntas impares para obtener el tipo de cohesión y haciendo lo propio con las preguntas pares para obtener el tipo de adaptabilidad. Esta fue procesada mediante una base de datos con la información cuantitativa, en el programa estadístico Excel v. 2007 para Windows Vista.

Se colocó la información en el orden del 1 al 42, según la información brindada por cada una de las madres. En la base de datos se transcribieron tanto los datos obtenidos con el instrumento como los datos obtenidos sobre la situación socioeconómica (Anexo 5).

Luego, con ayuda de la tabla de puntajes de Olson (Anexo 3 ), se relacionaron ambas dimensiones obteniendo el tipo de familia a la cual pertenecían y el tipo de funcionamiento que presentaban. Finalmente, se realiza la determinación de porcentajes de acuerdo al tipo de familia a la cual pertenecen en las dimensiones de cohesión y adaptabilidad respectivamente, $y$ al tipo de funcionamiento familiar que presentan en general.

\section{RESULTADOS}

Gráfico 1. Funcionamiento familiar según el modelo Circumplejo de Olson, en familias que tienen un niño con retardo mental en la institución educativa especial Divina Misericordia. Villa El Salvador - 2010

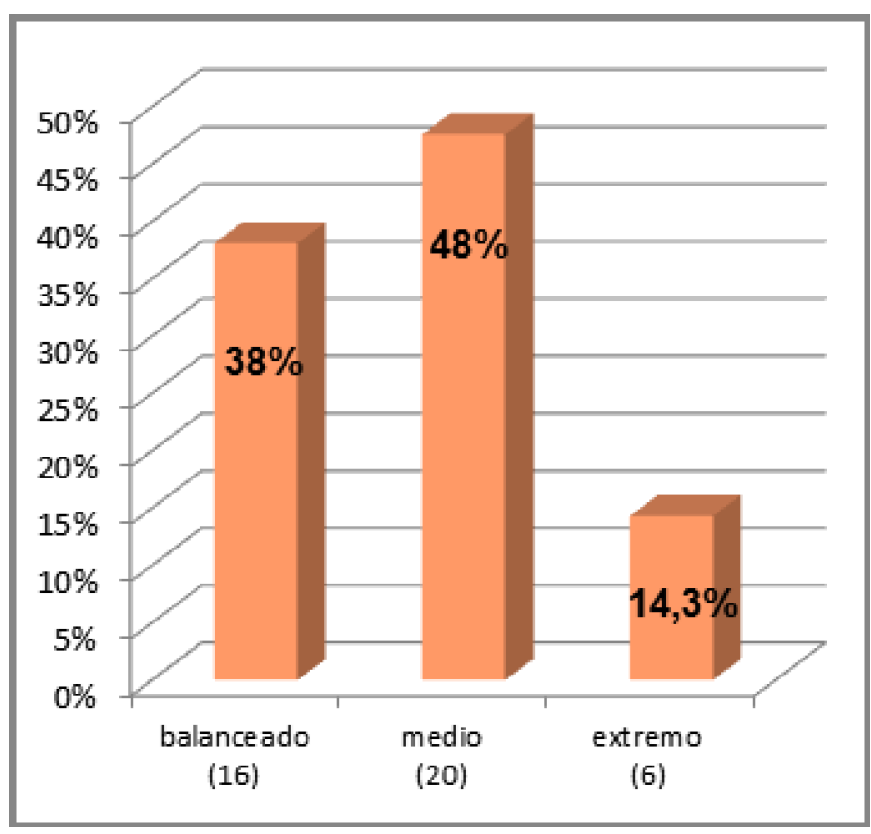

El Gráfico 1, muestra que de la población total 100\% (42 familias), el 48\% (13) de las familias con niños con retardo mental presenta un funcionamiento familiar de rango medio.

El Gráfico 2, muestra que en el funcionamiento familiar según cohesión el 38\% (16) de familias con niños con retardo mental pertenecen al tipo de familia semirrelacionada, teniendo un rango medio de cohesión.

El Gráfico 3, muestra que en el funcionamiento familiar según adaptabilidad el $42.9 \%$ (18) de familias con niños con retardo mental son altamente caóticas. 


\section{Gráfico 2. Funcionamiento familiar según cohesión, en familias que tienen un niño con retardo mental en la institución educativa especial Divina Misericordia. Villa El Salvador - 2010}

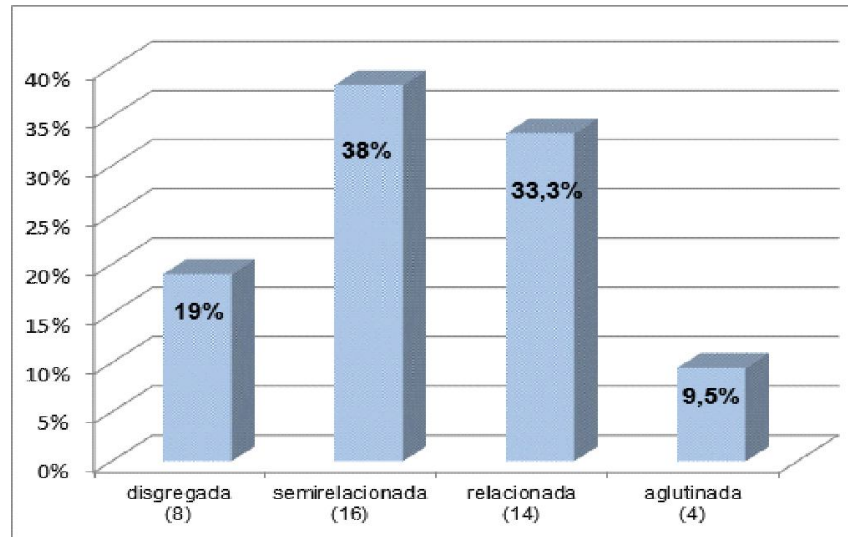

Gráfico 3. Funcionamiento familiar según adaptabilidad, en familias que tienen un niño con retardo mental en la institución educativa especial Divina Misericordia. Villa El Salvador - 2010

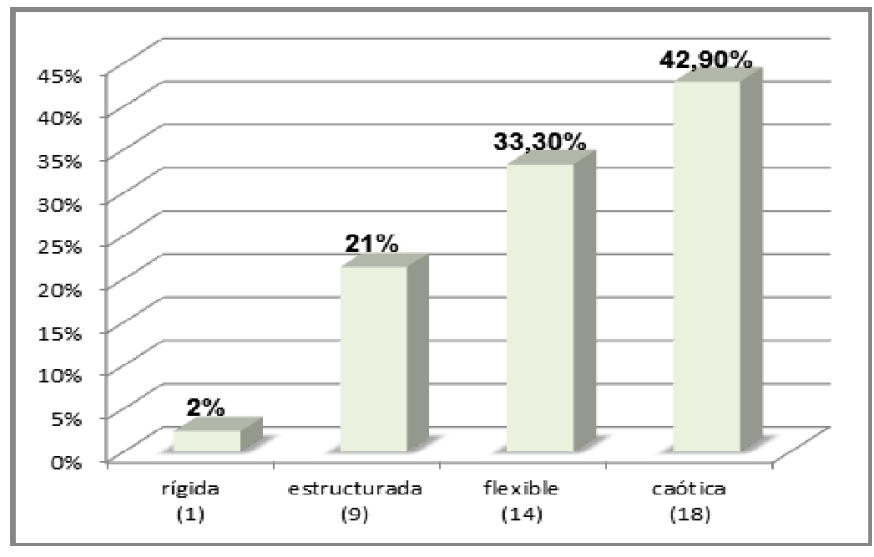

\section{DISCUSIÓN}

En el estudio se buscó determinar el funcionamiento familiar de familias que tienen un niño con retardo mental, y viven en Villa El Salvador y tienen un nivel socioeconómico medio bajo. Para ello, con el fin de visualizar integralmente el tipo de familia según su funcionamiento, se utilizó el Modelo Circumplejo de los Sistemas Familiares de Olson que estudió el funcionamiento familiar en tres dimensiones: cohesión, adaptabilidad y comunicación. Para efecto de este estudio sólo se analizaron las dos primeras dimensiones mencionadas anteriormente, según lo establecido por el modelo.

El resultado más relevante obtenido respecto al funcionamiento familiar es el rango medio, (Gráfico 1). Este tipo de funcionamiento familiar según la teoría de Olson no es saludable, debido a que tiene tendencia a la disfuncionalidad familiar. Estos resultados puede deberse a diversos facto- res como consecuencia de tener un niño con retardo mental, que afecta la relación conyugal, entre padres e hijos sin discapacidad, entre hermanos y la relación como padres.

Los padres, pilares de la familia, tienen muchos pensamientos y sentimientos de preocupación durante la crianza del niño con retardo mental, entre los que destacan están la parte económica, la situación del cuidado, educación, entre otros. En nuestra sociedad esto está enfocado en la parte económica, debido a que no cuentan con el dinero para pagar especialistas para la estimulación y educación del niño, sólo pueden acceder a instituciones educativas especiales que pertenecen al Ministerio de Educación en las cuales sólo pueden ayudar al niño en la parte educativa (17). Esta situación, probablemente, se encuentre presente durante el resto de sus vidas, debido a que el niño siempre será dependiente y necesitará del cuidado de otra persona; en algunos casos si el amor de la familia no es fuerte, los padres sienten «haber fallado»y deben enfrentar el repudio social y sentir vergüenza en vez de orgullo al mostrar la progenie. Significa fracasar en la necesidad de trascendencia y perder la seguridad en el futuro, ya que no sólo no van a recibir el apoyo de ese hijo cuando lleguen a la vejez, sino que tendrán la responsabilidad de cuidarlo y protegerlo por tiempo indefinido (9).

Respecto a la relación conyugal, en ésta pueden influir aspectos como los de la economía, amistades, toma de decisiones, trabajo y utilización de los tiempos libres, convirtiéndose en fuentes de conflicto ante el diagnóstico del hijo con retardo mental, porque tanto la madre como el padre pueden culparse por el problema que tiene el niño, generando separaciones o resentimientos entre ambos, experimentando rabia, temor, dolor e incredulidad por la situación que se está viviendo (25). Además, la exigente atención y cuidado que debe brindarse al niño puede conllevar un descuido de la pareja tanto en la parte sexual como en la afectiva, generando que en la mayoría de los casos sea el hombre el que busque pasar mayor tiempo en el trabajo o con los amigos evitando estar en casa(16). En los hogares donde existe el machismo y problemas de alcoholismo, para la madre el niño no es el único implicado en sus problemas también lo es el esposo, quien será posiblemente considerado el culpable de haber procreado un hijo en estado etílico (17).

Otro aspecto que puede estar influyendo en el resultado obtenido en el estudio, es el ingreso del niño a la etapa escolar y particularmente a un colegio especial. Uno de los padres tendrá que modificar drásticamente sus actividades cotidianas en función de las necesidades de aten- 
ción de su hijo, de tal manera que, a veces, es imposible cumplir con un horario de trabajo creando problemas insuperables causando que mayormente la madre deje de lado sus sueños o aspiraciones y se quede con un sentimiento de frustración sintiéndose infeliz. Este aspecto se puede agravar, si el ingreso económico de la pareja, más aún el sentir que es la única que tendrá que afrontar la situación durante casi todo el día. En las zonas urbanas marginales, aún las mujeres dependen económicamente de la pareja (18) y esto se ve reflejado en nuestra investigación, porque el $62 \%$ de las madres son amas de casa mientras que el $81 \%$ de los padres trabajan, situación que puede estar influyendo en la funcionamiento familiar (3).

Otro factor que puede estar influyendo en los resultados obtenidos es la relación entre padres e hijos sin discapacidad, puede estar a que los hermanos del niño con retardo mental pasan a un segundo plano, pueden sentirse excluidos de los nuevos procesos y roles familiar o culpable por haber escapado a la enfermedad. Los padres concentran casi toda la atención en el niño con necesidades especiales y probablemente están mucho más pendientes de su salud y de su educación, debido a que no es independiente como los otros niños. En algunos hogares, en cambio, puede ocurrir que la madre brinda más atención al niño con discapacidad intelectual y el padre pone toda la atención en los hijos sanos debido a que tiene todas las expectativas a futuro puestas en ellos. Esto puede ocasionar reclamos por parte de la madre lo cual conlleva a peleas constantes en la familia $(4,27)$.

Otro factor a considerar en los resultados es la relación entre hermanos. Probablemente los niños que no tienen el problema de discapacidad intelectual suelen experimentar sentimientos contradictorios que se encuentran entre la rabia y la pena. Algunos se sienten desorientados y avergonzados. Tener en la familia a un niño enfermo, desfigurado o incapacitado con secuelas neurológicas lo marca como diferente. Comúnmente los padres suelen dejar a los niños en casas de familiares o de amigos para evitar que se encuentren inmersos en la situación, lo cual puede causar falta de conocimiento de los problemas que se vive en la familia, creando desconcierto en ellos; tanto que pueden creer que los padres no tienen mucho tiempo para ellos, notando un cambio de ritmo en casa y en su vida cotidiana, quizás sin saber qué motivo lo ha causado. Cuando los otros hijos son adolescentes los sentimientos serán diferentes. Ellos pueden sentirse diferentes a su grupo de iguales debido a que tienen más responsabilidades (adquiridas o delegadas) que, a la vez, les pueden generar una sensación de sobrecarga $(1,4)$. También puede estar motivada por las renuncias que hayan tenido que hacer para cuidar de su hermano o por las preocupaciones que tienen (diferentes a las de los demás). Algunas veces se sienten solos debido a que no pueden compartir con el hermano con discapacidad muchas experiencias, ni tampoco pueden presentarlo con sus compañeros por vergüenza, lo que puede llevarlos posiblemente a un aislamiento, o por el contrario, a un exceso de actividad fuera de casa. Esto puede conducir a que los niños o adolescentes tomen actitudes de cólera o tristeza, lo que se verá reflejado en sus conductas, creando problemas y deteriorando un funcionamiento familiar saludable $(12,18)$.

Si comparamos los resultados obtenidos con otros estudios, el funcionamiento familiar, de rango medio encontrado en esta investigación, tiene semejanza con el obtenido en la investigación de Tejada y Grimaldo realizada en México en el 2005, la cual fue realizada en familias con perfil social similar al de nuestra investigación ellos señalan haber obtenido el mismo tipo de funcionamiento familiar, el cual fue determinado por serias dificultades de comunicación y comprensión entre los miembros de la familia, situando a los niños con retardo mental como demandantes de atención, sintiéndose abrumados por no saber cómo tratarlos y ayudarles con su enfermedad, lo cual provoca quiebre en las relaciones familiares (15).

Por el contrario, en la investigación de González y Herrera se identificó un funcionamiento familiar balanceado el cual se logró por una compenetración y ayuda mutua entre los miembros de la familia, logrando equilibrio y estabilidad familiar y generando un mejor ambiente de desarrollo para el niño con retardo mental (14).

Debido a los resultados encontrados en las investigaciones anteriores, no se debe dejar de mencionar el otro resultado relevante obtenido, fue que el $38,1 \%$ (10) de las familias presenta un nivel de funcionamiento familiar balanceado, encontrándose saludables, existiendo armonía, expresando los sentimientos y el afecto; además, la comunicación de sus miembros se cumple y los roles se desarrollan adecuadamente. Estos resultados nos dejan ver que en la sociedad actual también encontramos familias que tienen un niño con retardo mental y cada miembro desarrolla un rol óptimo dentro del funcionamiento familiar. Posiblemente, porque ya han logrado una adaptación a la realidad y a la situación obteniendo buenos lazos familiares y porque cada miembro de la familia desarrolló una actitud hacia el lado positivo de la situación (14). Además, también puede deberse a la edad del niño ya que el $59 \%$ de los padres tiene hijos de 8 a 11 años lo cual puede haberlos ayudado a encontrarse ya adaptados a la realidad (11). 
Otro aspecto estudiado en esta investigación es el tipo de funcionamiento familiar según la dimensión de cohesión, la cual evalúa el grado por el cual los miembros están conectados o separados del resto de la familia. Esta dimensión cuenta con 5 tipos de familias las cuales permiten determinar el tipo de funcionamiento familiar. En este caso obtuvimos que el 38\% (16) de las familias incluidas en la investigación, presentó cohesión media A (Gráfico 2), lo que representa a la familia de tipo semirrelacionada, que indica la existencia de una unión afectiva moderada entre los familiares, cierta interdependencia entre sus miembros, aunque con cierta inclinación a la independencia siendo una familia funcional media. Esto probablemente ocurre debido a que la familia mantiene una buena relación la mayoría del tiempo, generando que algunos tengan la necesidad de tener su propio espacio, en especial el padre y los hermanos lejos del niño con retardo mental, lo cual provocaría en un futuro el desequilibrio familiar como separación de los padres, y problemas relacionados con la conducta de los hermanos, entre otros. Este resultado podría deberse también a que las familias incluidas en el estudio, tienen o mantienen el amor familiar para que los lazos se mantengan fuertes. Esto se puede determinar debido a que el $71,5 \%$ de los padres incluidos en la investigación están casados o conviven (Anexo 5).

Al confrontar los resultados de nuestra investigación con otros estudios, similares resultados en la investigación de Ramírez y colaboradores en el 2001, Funcionalidad familiar y enfermedad crónica infantil, que el mayor porcentaje de familias pertenecía también al tipo de familia semirrelacionado y los niños tienen el mismo tipo de realidad familiar que los incluidos en esta investigación, teniendo padres casados, un nivel socioeconómico medio bajo y son cuidados por sus madres (13).

Otro aspecto estudiado en esta investigación es el tipo de funcionamiento familiar según la dimensión de adaptabilidad, que permite evaluar la habilidad de un sistema familiar para cambiar su estructura de poder, las relaciones de roles y las reglas de las relaciones, en respuesta al estrés situacional propio del desarrollo(12). Esta dimensión cuenta también con 5 tipos de familias las cuales permiten determinar el tipo de funcionamiento familiar. En este caso obtuvimos que el 42,9\%(18) de las familias resultaron altamente caóticas. (Gráfico 4). En la familia de tipo caótico se evidencia alteraciones en la dinámica familiar, los roles de cada miembro no son claros, causando frecuentes cambios en las reglas que se hacen cumplir inconsistentemente, y las decisiones parentales son impulsivas. Esto puede ocurrir probablemente por la ausencia paterna debido al trabajo, encargándose del hogar solamente la madre, lo que causa una sobrecarga de actividades y roles, lo que puede generar que los hijos, incluyendo el niño con retardo mental, no cumplan con las decisiones por sentirse excluidos. También la madre siente sobrecarga de roles, no puede darse un tiempo para descansar u ocuparse de ella ya que no tiene quien la apoye con el cuidado del hogar y de los niños. Además, por la situación económica es imposible utilizar dinero para otras actividades como paseos, pagar una niñera, etc. El padre presenta una sobrecarga de estrés probablemente por el exceso de trabajo o por las preocupaciones económicas, lo cual causaría que comiencen las discusiones en la pareja, los malos tratos hacia los otros hijos, aumentando las probabilidades de que un padre u otro cuidador maltraten al menor. Contribuyen a esta acción las expectativas poco realistas sobre el desarrollo del niño, el escaso control de los impulsos, el estrés y el aislamiento social (20). Esto lleva a que inclusive el niño con retardo mental tenga malas conductas así como también las tienen los hermanos, convirtiéndose en un ciclo vicioso, generando deterioro en la adaptabilidad y disfuncionalidad familiar(12).

Los resultados de adaptabilidad de nuestra investigación son semejantes a los del estudio de Ramírez y colaboradores realizado en el 2001, Funcionalidad familiar y enfermedad crónica infantil, en el cual se obtuvo como resultado que en su mayoría (25\%), las familias tenían Adaptabilidad caótica (14), generada por razones similares a las especificadas anteriormente.

Los resultados obtenidos en el estudio muestran que actualmente, en nuestra sociedad hay un porcentaje (48\%) de familias que tienen un niño con retardo mental, que no reflejan una interacción dinámica entre los miembros de la familia, pero también existe un porcentaje de familias (38\%), que tienen una mejor relación y convivencia entre sus miembros apoyando entre todos al cuidado del niño especial $(3,19)$.

\section{REFERENCIAS BIBLIOGRÁFICAS}

1. Arés, P. Introducción a la Psicología de la familia. En: Colectivo de autores. Selección de lectura sobre psicología de las edades y la familia. La Habana: Editorial Ciencias Médicas; 2007.

2. López M, Riu M, Forner L. Cuidados Enfermeros. 1era ed. España. Elsevier; 2005.

3. Garza T. Trabajo con familias. 2da. ed. México. Universidad Autónoma de Nuevo León; 2009.

4. Polaino-Lorente A, Martínez Cano P. Evaluación psicológica y psicopatológica de la familia. 3ra ed. 
España. Editorial Universidad de Navarra - Instituto de Ciencias para la Familia; 2006.

5. Ministerio de Salud. Casos de Retardo Mental registrados en consulta externa 2005-2008. Informe de registro diario HIS. Oficina General de Estadística e Informática. Lima-Perú 2009.

6. Perales, A. ed. Manual de Psiquiatría «Humberto Rotondo». 3era ed. Perú. Editorial Universidad Nacional Mayor de San Marcos; 2008.

7. Johon R. Familias, Enfermedad y discapacidad. Barcelona. Editorial Gedisa; 2006.

8. Caballero Muñoz E, Fernández Díaz P. Director: Antonio José Ibarra Fernández. Tratado de Enfermería en Cuidados Críticos Pediátricos y Neonatales. Se ha culminado el Tratado en: Versión ON-LINE. ISSN: 1885-7124, Versión DVD. ISBN: 84-64-2447-2. Editado el 20 de octubre del 2006. Sección 10, capitulo 146.

9. Wilson, D. Manual de enfermería Pediátrica de Wong. 7ma ed. España. McGraw-Hill; 2009.

10. Asociación Americana sobre Retraso Mental AAMR. Retraso mental: Definición, clasificación y sistemas de apoyo. 10ma ed. EE.UU. Editorial F.A; 2007.

11. Olson D, Russell C, dSprenkle D. Circumplex model: systemic assessment and treatment of families. 1era Ed. EE.UU. Editorial Routledge; 1989.

12. Gafo J, Pérez J. Deficiencia mental y familia. 3era ed. Madrid, España. Editorial Universidad Pontificia de Comillas; 2006.

13. Ramírez Lumbreras C, Méndez Espinoza E, Barrón Garza F, Riquelme Heras H, Cantú Leyva R. Funcionalidad Familiar y Enfermedad Crónica Infantil. [Internet] México: Institución Nuevo Amanecer, 2003 [Citado 13 May 2009]. Disponible en: http:// www.nuevoamanecer.edu.mx/imgs/pdf/ Funcionalidadfamiliar.pdf
14. Pérez Martínez V, Lorenzo Parra Z. El impacto del déficit mental en el ámbito familiar. Rev Cubana Med Gen Integr. 2007; 23(3): 6-10.

15. Tejada Tayabes, L. Grimaldo Moreno, B. Maldonado Rodríguez, O. Percepciones de enfermos diabéticos y familiares acerca del apoyo familiar y el padecimiento. Rev Respyn. 2006; 7(1): 7-11.

16. Mendoza A. Guía metodológica para la intervención preventiva con familias. Lima: DEVIDA; 2006.

17. Ponce Ribas, A. Apoyando a los hermanos: Tres propuestas de intervención con hermanos de personas con discapacidad intelectual. 1era ed. Madrid Colección. FEAPS; 2006.

18. Verdugo, M (Ed.) Familias y discapacidad intelectual. 2da ed. Madrid.Colección FEAPS; 2006.

19. Amat C.M,y León Guevara. Salud comunitaria y familiar en el Perú. Fondo Editorial. Centro de investigación $1^{\circ}$ Edición. 2008.

20. Organización Panamericana de la Salud Organización Mundial de la Salud. La familia y la salud. Consejo Directivo $44^{\circ}$ Sesión del Comité Regional. Washington D.C.: 22 al 26 de septiembre del 2006. Disponible en: http://www.paho.org/spanish/ gov/cd/cd44-10-s.pdf

\section{Correspondencia}

Pedro Ferrer Honores

Urb. Pachacamac, Villa El Salvador.

Teléf. 2874844

Correoelectrónico: pafh-2402@hotmail.com

Forma de citar este artículo: Ferrer-Honores Pedro, MiscánReyes Ana, Pino-Jesús María, Pérez-Saavedra Vilma. Funcionamiento Familiar según el Modelo Circunplejo de Olson en Familias con un Niño que presenta retardo Mental. Rev enferm Herediana. 2013;6(2):51-58. 